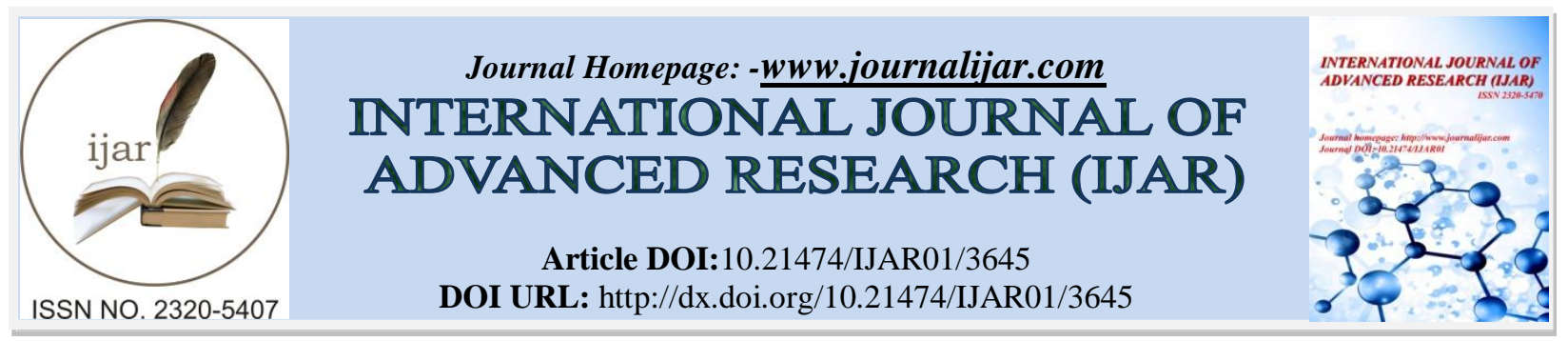

RESEARCH ARTICLE

\title{
COMPARATIVE STUDY ON TUNING METHODSFOR SISO SYSTEM.
}

\section{Manju Sharmila.N, MonishaSrihari and Nivethitha Devi .M.}

Department of Electronics and Instrumentation Engineering, St.Joseph's College of Engineering, Chennai-600119, Tamilnadu, India.

\section{Manuscript Info}

Manuscript History

Received: 09 January 2017

Final Accepted: 04 February 2017

Published: March 2017

Key words:-

IMC-PID, Direct Synthesis, SIMC, Setpoint tracking and Disturbance Rejection.

\section{Abstract}

This paper presents various tuning methods for a first order process with time delay. The performance of IMC based PID controller is compared with other tuning methods like Modification of IMC-PID for disturbance rejection, Direct synthesis, Direct Synthesis with disturbance rejection (DS-d) and Simple Internal Model Control (SIMC).This work analyses the various parameters of the system. The comparative study of set point tracking and disturbance rejection of a first order process is evaluated using performance criteria like ISE and IAE. From the comparison it was found that IMC-PID provides better results for set-point tracking as well as for the disturbance rejection.

Copy Right, IJAR, 2017,. All rights reserved.

\section{Introduction:-}

The Proportional-integral-derivative (PID) controllers are extensively used in the industrial applications at the regulatory level. The fundamental reason for choosing PID controllers is their relatively simple structure, which can be readily understood and allows them to easily resolve in the real world. However, it is observed that many PID controllers are not properly tuned and exertion has been made to systematically resolve this problem. Therefore, the goal of this work is, to develop a direct approach tuning method of controller from closed loop set point and comparisons has been made for different tuning methods [1].

There are certain goals for tuning methods. The tuning rules should be transparent and easy to memorize. The tuning rules should work on ample range of process. The tuning rules should be well motivated, and preferably modelbased and analytically derived.

There are different types of PID controller tuning techniques and they are based on open-loop or closed-loop plant tests and most of these techniques utilize the process gain $(\mathrm{k})$, time constant $(\tau)$ and time delay $(\theta)$. The vast article on PID controllers includes a wide variety of design and tuning methods based on the performance criteria. The two most oldest and common method of tuning are Zeigler-Nichols (ZN) [2] and Cohen and Coon[3]. These methods were developed to provide a closed-loop response with a quarter decay ratio. The ZN tuning method yields a very good disturbance response for integrating processes[4], but in another way, it results in rather aggressive settings and also give poor performance for process with a dominant delay.

The popular tuning methods for PID controllers which have been used for different kind of processes are Direct Synthesis (DS) [5] and Internal Model Control [6]. The output response based on both the approaches has satisfactory performance and robustness. The main purpose of using IMC-PID is that, it results in a controller with a 
single tuning parameter $(\lambda)$. For s system which is "minimum phase", $\lambda$ is equivalent to a closed loop time constant the "speed of response" of the closed-loop system. IMC-PID provides satisfactory response for set point tracking but it provides poor disturbance rejection for higher order processes in order to overcome this various disturbance rejection techniques were proposed. In the Direct Synthesis (DS) method, the controller design is based on a desired closed-loop transfer function. The next step in the controller is calculated analytically so that the closed-loop setpoint matches the desired response. The apparent benefit of using direct synthesis is that the performance requirements are fused directly through the closed loop transfer function. Therefore, the resulting direct synthesis controllers contribute to achieve well for set-point changes. The disturbance rejection is considered to be important so different modification techniques were proposed.

The modification proposed by Dan Chen and Dale E. Seborget al for disturbance rejection of Direct synthesis(DSd)[7] does not provide satisfactory results. The modification for IMC-PID[8] proposed by Shamsuzzoha for disturbance rejection[9] could not produce the expected results.

SKOGESTAD [10] has altered the integral time in SIMC method which is an exceptional remedy for processes with a large time constant [11]in order to improve load disturbance rejection.

Although the PID tuning rule on the basis of IMC and direct synthesis methods gives excellent performance for setpoint changes, it shows slow output responses to input (load) disturbances for lag-dominant as well as integrating processes.

Therefore the goal of this paper is to find the IMC-PID procedure for set-point tracking and disturbance rejection to the processes. In this paper, first-order with time delay has been considered and comparisons have been made against Direct Synthesis method, IMC-PID, direct synthesis disturbance rejection (DS-d),modified IMC(Shamsuzzoha) and SIMC method. The various parameters are analysed to obtain the satisfactory results for set point tracking and disturbance rejection.

\section{IMC-PID:-}

Internal Model Control based PID controller has the advantage of good set point tracking when it is compared with other methods. The fig 1 shows the basic IMC structure

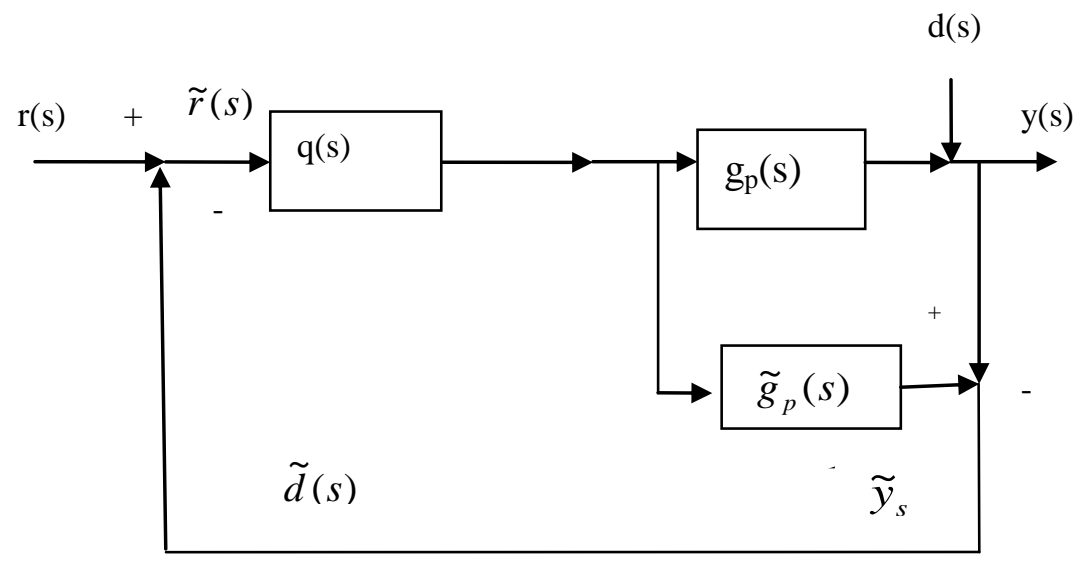

Fig 1:- IMC Structure

It is done by padè approximation technique or using Taylor's series. In this paper, we have used only the padè approximation technique. IMC-PID provides poor disturbance rejection in order to improve this SIMC have been developed because it provides a better disturbance rejection.

IMC-Based PID Design For A First-Order+ Deadtime Process:-

Consider a first order process with dead time 


$$
g_{p}(s)=\frac{k e^{-\theta s}}{\tau s+1}
$$

Where $\mathrm{k}, \tau$ and $\theta$ are the process gain, time constant and time delay.

Step 1: Use a first order padè approximation for the dead time.

$e^{-\theta s}=\frac{-0.5 \theta s+1}{0.5 \theta s+1}$

The main reason for using padè approximation is to obtain the simple PID control structure with enhanced performance.

$\tilde{g}_{p}(s)=k\left(\frac{-0.5 s+1}{(0.5 s+1)(\tau s+1)}\right)$

Separate $\tilde{g}_{p}(s)$ into invertible and non-invertible parts

$$
g_{p^{-}}(s)=k\left(\frac{1}{(0.5 s+1)(\tau s+1)}\right)
$$

$$
g_{p+}(s)=(-0.5 s+1)
$$

Where $g_{p+}(s)$ and $g_{p}(s)$ are the non-inverted and inverted parts of the model, respectively.

Step 2:Find the feedback controller

$q(s)=g_{p-}^{-1}(s) * f(s)$

Where $f(s)=\frac{1}{(\lambda s+1)^{n}} ; \mathrm{n}=1$ (for first order)

$$
q(s)=\frac{[(0.5 s+1)(\tau s+1)]}{k(\lambda s+1)}
$$

Where $\lambda$ is the filter tuning parameter.

Step 3: The filter $\mathrm{f}(\mathrm{s})$ is added andthe numerator term to find the PID equivalent,

$g_{c}(s)=\frac{\left[0.5 \tau \theta s^{2}+(\tau+0.5 \theta) s+1\right]}{k(\lambda+0.5 \theta)}$

Comparing with the standard expression $g_{c}(s)=k_{c} \frac{\tau_{I} \tau_{D} s^{2}+\tau_{I} s+1}{\tau_{I} s+1}$ we can calculate the values of $\mathrm{k}_{\mathrm{c}}, \tau \mathrm{I}$ and $\tau_{\mathrm{D}}$

$$
\begin{aligned}
& k_{c}=\frac{2 \tau+\theta}{2 k(\lambda+\theta)} \\
& \tau_{I}=\tau+0.5 * \theta_{\tau_{D}}=\frac{\tau \theta}{2 \tau+\theta}
\end{aligned}
$$

Rivera et al. (1986) recommend that $\lambda>0.8 \theta$ because of the model uncertainty due to the padè approximation.

\section{MODIFIED IMC-PID (Shamsuzzoha):-}

The modified IMC proposed by Shamsuzzoha has the same way of solving as IMC-PID. The variation is only in terms of the tuning parameter. Here we substitute the tuning parameter by 0.6 times of $\theta$.

$$
\begin{aligned}
& \lambda=0.6 \theta \\
& k_{c}=\frac{2 \tau+\theta}{3.2 k \theta} \\
& \tau_{I}=\min \{(\tau+0.5 \theta), 4.8 \theta\}
\end{aligned}
$$




$$
\tau_{D}=\frac{\tau \theta}{2 \tau+\theta}
$$

\section{Direct Synthesis:-}

In the direct synthesis method, the design of the controller is based on the closed loop transfer function. It can be done by either padè approximation or Taylor series

Consider a first order system with the dead time

$$
\tilde{G}=\frac{k_{p} e^{-\theta_{p} s}}{\tau_{p} s+1}
$$

The exponential term can be approximated using padè approximation and Comparing with the standard form we get the desired parameters as for PID controller as:

$$
\begin{aligned}
K_{c} & =\frac{\tau_{p}+0.5 \theta_{p}}{k_{p}\left(\tau_{c}+\theta_{p}\right)} \\
\tau_{I} & =\tau_{p}+0.5 \theta_{p} \\
\tau_{D} & =\frac{\tau_{p} \theta_{p}}{2 \tau_{p}+\theta_{p}}
\end{aligned}
$$

\section{Direct Synthesis Disturbance Rejection (Ds-d):-}

Consider a first order system with delay

$G_{p}(s)=\frac{k e^{-\theta s}}{\tau s+1}$

The standard form for direct synthesis for a first order system with delay is given by

$$
G_{p}(s)=\frac{\frac{k e^{-\theta s}}{\tau s+1}}{1+\frac{k e^{-\theta s}}{\tau s+1} k_{c}\left(1+\frac{1}{\tau_{I} s}+\tau_{D} s\right)}
$$

The time delay term in the denominator can be approximated by using a first order pade approximation,

$e^{-\theta s} \approx \frac{1-0.5 \theta s}{1+0.5 \theta s}$

We use pade approximation techniques to obtain the $\mathrm{G}_{\mathrm{p}}(\mathrm{s})$, it is then compared with the standard expression to obtain the various parameters $\mathrm{k}_{\mathrm{c}}, \tau_{\mathrm{I}}$ and $\tau_{\mathrm{D}}$.

By comparing with the standard form, the Parameters obtained are,

$$
\begin{aligned}
& K_{c}=\frac{1}{k} \frac{\left(2 \tau \theta+\frac{\theta^{2}}{2}\right)\left(3 \tau_{c}+\frac{\theta}{2}\right)-2 \tau_{c}^{3}-3 \tau_{c}^{2} \theta}{2 \tau_{c}{ }^{3}+3 \tau_{c}{ }^{2} \theta+\frac{\theta^{2}}{2}\left(3 \tau_{c}+\frac{\theta}{2}\right)} \\
& \tau_{1}=\frac{\left(2 \tau \theta+\frac{\theta^{2}}{2}\right)\left(3 \tau_{c}+\frac{\theta}{2}\right)-2 \tau_{c}{ }^{3}-3 \tau_{c}^{2} \theta}{(2 \tau+\theta) \theta}
\end{aligned}
$$




$$
\tau_{D}=\frac{3 \tau_{c}{ }^{2} \tau \theta+\frac{\tau \theta^{2}}{2}\left(3 \tau_{c}+\frac{\theta}{2}\right)-2(\tau+\theta) \tau_{c}{ }^{3}}{\left(2 \tau \theta+\frac{\theta^{2}}{2}\right)\left(3 \tau_{c}+\frac{\theta}{2}\right)-2 \tau_{c}{ }^{3}-3 \tau_{c}{ }^{2} \theta}
$$

\section{Simple Internal Model Control (Skogestad):-}

Skogestad Internal Model Control is the modified method of IMC-PID for improved disturbance rejection than IMC-PID. Here the only parameter used is $\tau_{\mathrm{c}}$. For improved disturbance rejection and good robustness the tuning parameter should be made equal to the value of time delay $(\theta)$. Here we consider a first order process with delay and the parameters are obtained using the Half reduction rule[9]

The largest neglected time constant in the denominator is evenly distributed to the effective delay and the smallest retained time constant.

$$
G(S)=\frac{\pi_{j}\left(-T_{j 0} s+1\right)}{\pi_{i}\left(\tau_{i 0} s+1\right)} e^{-\theta s}
$$

Let the original model be

$$
G(s)=\frac{1}{\tau_{1} s+1} e^{-\theta s}
$$

Where

$$
\begin{gathered}
\tau_{1}=\tau_{10}+\tau_{20} / 2 \\
\theta=\theta_{0}+\tau_{20} / 2+\sum_{i \geq 3} \tau_{i 0}+\sum_{j} T_{j 0}+\frac{h}{2}
\end{gathered}
$$

The delay term is approximated by using Half Rule and from the reduced order of the system the various parameters can be calculated and the PID controller is designed.

$$
\begin{aligned}
& K_{C}=\frac{1}{K} \frac{1}{\tau_{C}+\theta} \\
& \tau_{I}=\min \left(\tau, 4\left(\tau_{c}+\theta\right)\right)
\end{aligned}
$$

\section{Results and Discussion:-}

The transfer function considered for our work is $g_{p}(s)=\frac{e^{-s}}{0.2 s+1}$

\section{Set point tracking:-}

Table 1 shows the results obtained for set point tracking for various methods for the above transfer function:

By analyzing the table1, we can see that the settling time is less for IMC-PID when compared with modified IMC, Direct synthesis, Direct synthesis-d and SIMC. Therefore the IMC-PID shows a good set point tracking when compared to other methods. This can also be found from the fig 2 .

The fig1 shows the response obtained for the transfer function $g_{p}(s)=\frac{e^{-s}}{0.2 s+1}$ using various methods to track the set point. The response shows that IMC settles faster when compared to modified IMC, Direct synthesis, direct synthesis-d and SIMC. The settling time varies depending on the tuning parameter $\lambda$ or $\tau_{\mathrm{c}}$. The errors were found to be less in IMC-PID method compared to modified IMC, Direct synthesis, direct synthesis-d and SIMC. 
Table 1:-Comparison of various tuning method for Set point tracking.

\begin{tabular}{|l|l|l|l|l|l|l|l|}
\hline & Settling Time & $\mathbf{K}_{\mathbf{c}}$ & $\boldsymbol{\tau}_{\mathbf{I}}$ & $\boldsymbol{\tau}_{\mathbf{D}}$ & ISE & IAE & $\boldsymbol{\tau}_{\mathbf{C}}$ or $\boldsymbol{\lambda}$ \\
\hline IMC & 8 & 0.5185 & 0.700 & 0.1429 & 1.12 & 1.378 & 0.850 \\
\hline IMC-Mod & 10 & 0.4375 & 0.700 & 0.1429 & 1.193 & 1.601 & 0.600 \\
\hline DS & 15 & 0.280 & 0.700 & 0.1429 & 1.614 & 2.501 & 1.500 \\
\hline DS-d & 13 & 0.3179 & 0.665 & 0.1095 & 1.46 & 2.097 & 0.380 \\
\hline SIMC & 10 & 0.100 & 0.200 & 0.00 & 3.471 & 4.486 & 1.00 \\
\hline
\end{tabular}

In Fig 1, The time taken for the IMC-PID to settle is less when compared to the modified IMC-PID. The errors are also found to be lesser.

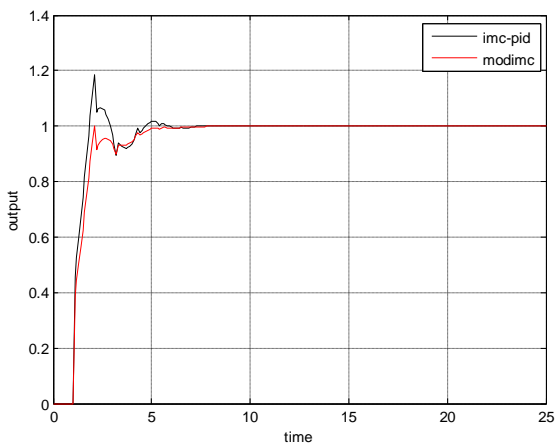

Fig 1:-

In Fig 2, Direct Synthesis provides good set point tracking but when compared with IMC-PID for first order system with dead time performance

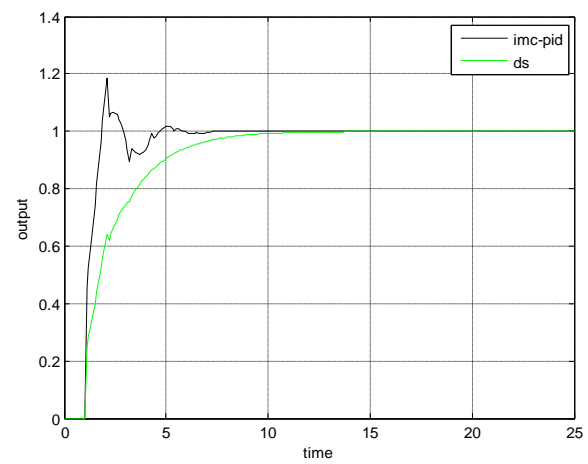

Fig 2:-

In Fig 3, the performance is evaluated by comparing Direct Synthesis for disturbance In rejection with IMC-PID. It is clear that IMC-PID provides good set point tracking.

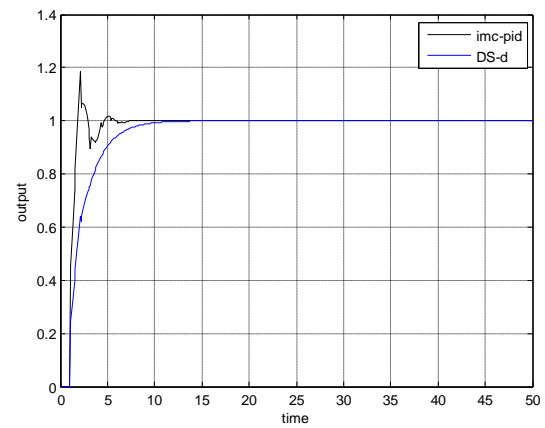

Fig 3:- 
In Fig 4, It is clear from the graph that setting time is less for IMC-PID than SIMC.

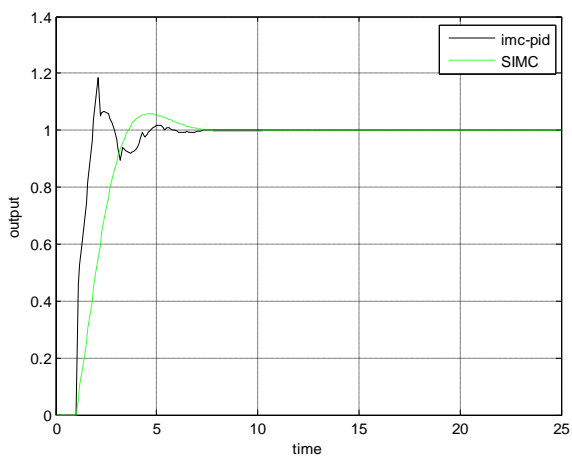

Fig 4:-

\section{Disturbance Rejection:-}

The table 2 shows the improved disturbance rejection for the transfer function $g_{p}(s)=\frac{e^{-s}}{0.2 s+1}$ using various methods to obtain the improved disturbance rejection.

The fig 5-8 shows the response obtained for the transfer function $g_{p}(s)=\frac{e^{-s}}{0.2 s+1}$ using various methods for disturbance rejection. The response shows that IMC-PID shows a good set point tracking and provides better disturbance rejection compared to other methods.

Table 2:- Comparison of various tuning methods for Disturbance Rejection.

\begin{tabular}{|c|c|c|c|c|c|c|c|}
\hline & Settling Time & $\mathbf{K}_{\mathbf{c}}$ & $\boldsymbol{\tau}_{\mathbf{I}}$ & $\boldsymbol{\tau}_{\mathbf{D}}$ & ISE & IAE & $\boldsymbol{\tau}_{\mathbf{C}}$ or $\boldsymbol{\lambda}$ \\
\hline IMC & 9 & 0.5185 & 0.700 & 0.1429 & 2.408 & 3.033 & 0.850 \\
\hline IMC-Mod & 8 & 0.4375 & 0.700 & 0.1429 & 2.510 & 3.200 & 0.600 \\
\hline DS & 14 & 0.280 & 0.700 & 0.1429 & 3.218 & 4.808 & 1.500 \\
\hline DS-d & 12 & 0.3179 & 0.665 & 0.1095 & 2.920 & 4.194 & 0.380 \\
\hline SIMC & 8 & 0.100 & 0.200 & 0.00 & 1.736 & 2.243 & 1.00 \\
\hline
\end{tabular}

In Fig 5, It is clear from the graph that disturbance rejection is better for IMC-PID than modified IMC-PID.

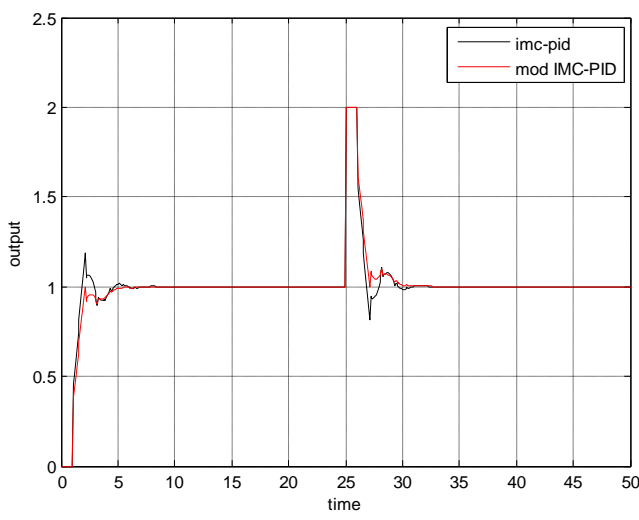

Fig 5:- 
In Fig 6, The performance is evaluated by comparing Direct Synthesis with IMC-PID. It is clear that IMC-PID provides good disturbance rejection.

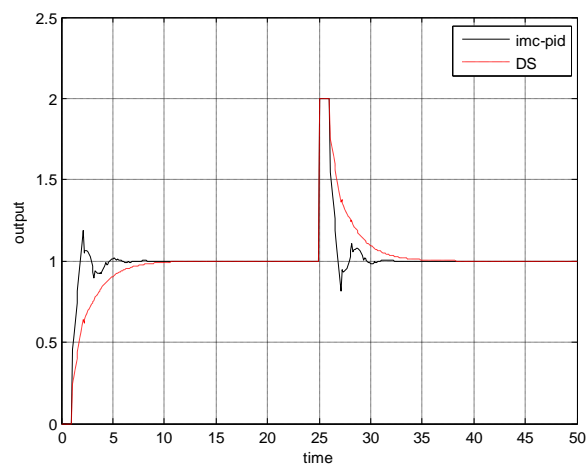

Fig6:-

In Fig 7, The graph depicts that the IMC-PID shows better result for disturbance rejection compared to DS-d.

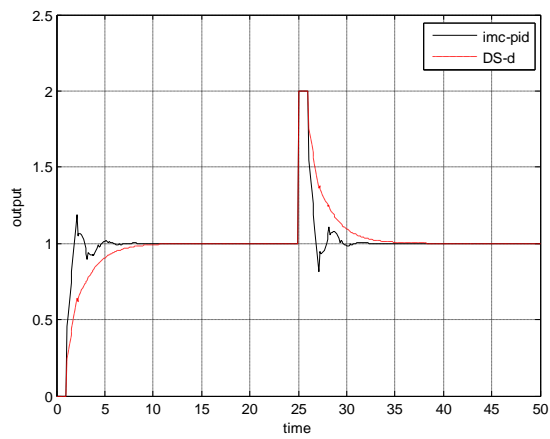

Fig 7:-

In Fig 8,For the first order with time delay process, the IMC-PID shows better result for disturbance rejection compared to SIMC.

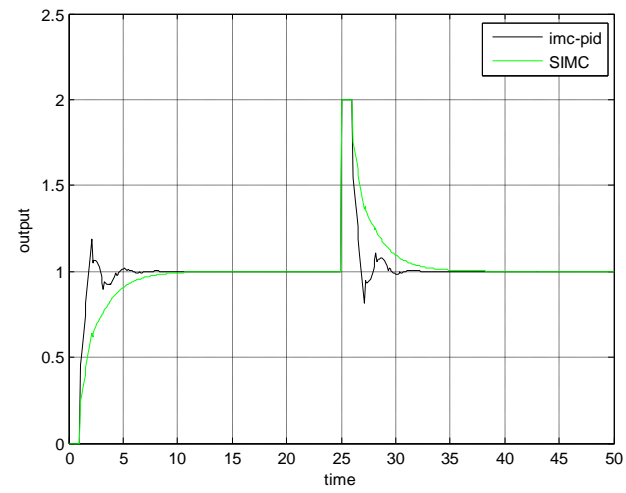

Fig 8:-

\section{Conclusion:-}

The IMC-PID provides good set point tracking and disturbance rejection considering the above first order with time delay process when compared to other methods. The DS methods are generally dependent upon the desired closedloop transfer function for the set-point changes. Subsequently, the resulting Direct Synthesis controllers tend to perform well for the set-point changes in comparison to the disturbance rejection where the result is not satisfactory. Hence, controller design that produces satisfactory results for the disturbance rejection compared to the set-point tracking is considered as a major design problem. Therefore, it is withal to develop a modified direct synthesis 
approach based on disturbance rejection. The new design method will be denoted by "DS-d".Another method for disturbance rejection of IMC-PID was proposed by Mohammad Shamsuzzoha. This method does not produce satisfactory results. Another method was proposed by SigurdSkogestad known as SIMC. This method provides improved disturbance rejection for the higher order process.

\section{Reference:-}

1. Wen Tan, Jizhen Liu, Tongwen Chen, Horacio J. Marquez, "Comparison of some well-known PID tuning formulas", Department of Automation, North China Electric Power University, Zhuxinzhuang, Dewai, Beijing 102206, PR China,Department of Electrical \& Computer Engineering, University of Alberta, Edmonton, AB, Canada T6G 2V.

2. Ziegler, J. G.; Nichols, N. B. Optimum Settings for Automatic Controllers. Trans. ASME 1943, 65, 433.

3. Poorvi Jain and Pramod Kumar Jain, "Design Of FOPDT PID Controller By Different Tuning Methods For Industrial Process Control", M.Tech Scholar, Electronics \& Instrumentation Engineering Department, Shri G.S.I.T.S, Indore, India. Associate Professor, Electronics \& Instrumentation Engineering Department, Shri G.S.I.T.S, Indore, India

4. G.Madhu Kumar, V. Suma Deepthi, "Design and Development of IMC Tuned PID Controller for Disturbance Rejection of Pure Integrating Process",PG Scholar, Malla Reddy Engineering College, India, Assistent Professor, Malla Reddy Engineering College, India

5. Ms. Dighe Y, Prof. Kadu C and Prof. Parvat B, "Direct Synthesis Approach for Design of PID Controller", in International Journal of Application or Innovation in Engineering \& Management (IJAIEM).

6. Daniel E. Rivera, Manfred Morari, and SigurdSkogestad, " Internal Model Control. 4. PID Controller Design", Chemlcal Engineering, 206-4 1, California Institute of Technology, Pasadena, California 91125.

7. Dan Chen and Dale E. Seborg, "PI/PID Controller Design Based on Direct Synthesis and Disturbance Rejection”, Department of Chemical Engineering, University of California, Santa Barbara, California 93106.

8. Mohammad Shamsuzzoha, "IMC based robust PID controller tuning for disturbance rejection", Department of Chemical Engineering, King Fahd University of Petroleum and Minerals, Dhahran 31261, Saudi Arabia Central South University Press and Springer-Verlag Berlin Heidelberg 2016.

9. Shamsuzzoha M, Lee M, "IMC-PID controller design for improved disturbance rejection of time-elayed processes [J]", IndEngChem Res, 2007.

10. SigurdSkogestad, "Simple analytic rules for model reduction and PID controller tuning", Department of Chemical Engineering, Norwegian University of Science and Technology, N-7491Trondheim, Norway.

11. Jietae Lee, Wonhui Cho, and Thomas F. Edgar, "Simple Analytic PID Controller Tuning Rules Revisited", Department of Chemical Engineering, Kyungpook National University, Taegu 702-701, Korea, enGibbs, A-503 Shin Young Gwell Estates, Bansong-dong, 93-10, Hwasung, Gyeonggi 445-160, Korea, Department of Chemical Engineering, University of Texas, Austin, Texas 78712, United States.

12. Ankit K. Shah, MarkanaAnilkumar and Nishant Parikh, " Performance analysis of IMC based PID controller tuning on approximated process model", Nirma University Journal of engineering and technology

13. SigurdSkogestad_, "Probably the best simple PID tuning rules in the world", Department of Chemical Engineering, Norwegian University of Science and Technology, N-7491 Trondheim Norway

14. Ibrahim Kaya, "IMC based automatic tuning method for PID controllersin a Smith predictor configuration", Department of Electrical and Electronics Engineering, Faculty of Engineering, Inonu University, 44069 Malatya, Turkey.

15. Rames C Panda, "Synthesis of PID Controller for unstable and integrating process", Department of Chemical Engineering, CLRI(CSIR), Adyar, Chennai 600020, India.

16. M. Shamsuzzoha and Moonyong Lee (2012). IMC Filter Design for PID Controller Tuning of Time Delayed Processes, PID Controller Design Approaches - Theory, Tuning and Application to Frontier Areas, Dr.MarialenaVagia (Ed.), ISBN: 978-953-51-0405-6

17. Horn I. G, Arulandu J. R, Christopher J. G, Van Antwerp J.G and Braatz R.D, (1996), "Improved Filter Design in Internal Model Control", Ind. Eng. Chem. Res, Vol.35, pp.3437-3449

18. Skogestad S, Grimholt C, "The SIMC method for smooth PID controller [C]// PID Control in the Third Millennium, Advances in Industrial Control", Springer, 2012

19. Ian G. Horn, Jeffery R. Arulandu, Christopher J. Gombas, Jeremy G. VanAntwerp, and Richard D. Braatz, "Improved Filter Design in Internal Model Control", Department of Chemical Engineering, University of Illinois at UrbanasChampaign, 600 South Mathews Avenue, Box C-3, Urbana, Illinois 61801-3792. 
20. M. Parada, D. Sbarbaro, R. A. Borges \& P. L. D. Peres, "Robust PI and PID design for first- and secondorder processes with zeros, time-delay and structured uncertainties"

21. Ruscio D. D, (2010), "On Tuning PI Controllers for Integrating PlusTime Delay Systems, Modelling, Identification and Control", Vol.31, pp.145-164.

22. Seborg D. E, Edgar T. F, Mellichamp D. A, Doyle F. J, (2010), "Process Dynamics and Control", $3{ }^{\text {rd }}$ ed, John Wiley \& Sons: New York.

23. Grimholt C, Skogestad S, (2012), "Presented optimal PI-control and verification of the SIMC Tuning Rule", IFAC conference on advances in (PID'12), Brescia, Italy. 